Supporting Information for Organic Letters

\title{
A Fluorinated Selenide Linker for Solid-Phase Synthesis of $n$-Pentenyl Glycosides
}

\author{
Mickael Mogemark, ${ }^{\dagger}$ Linda Gustafsson, ${ }^{\dagger}$ Christoffer Bengtsson, ${ }^{\dagger}$ Mikael \\ Elofsson, ${ }^{\dagger *}$ and Jan Kihlberg ${ }^{\dagger * * *}$ \\ 'Organic Chemistry, Department of Chemistry, Umeå University, SE-901 87 Umeå, \\ Sweden and "AstraZeneca R\&D Mölndal, SE-431 83 Mölndal, Sweden. \\ mikael.elofsson@chem.umu.se, jan.kihlberg@chem.umu.se
}

General methods and materials. All reactions were carried out at room temperature under an inert nitrogen atmosphere using dry, freshly distilled solvents. TLC was performed on Silica Gel $\mathrm{F}_{254}$ (Merck) and detection was carried out by examination under UV light and by charring with 10\% sulfuric acid. Flash column chromatography was performed on Silica Gel (Matrex, $60 \AA, 35-70 \mu \mathrm{m}$, Grace Amicon). ${ }^{1} \mathrm{H}$ and ${ }^{13} \mathrm{C}$ NMR spectra were recorded at 400 or $500 \mathrm{MHz}$ for solutions in $\mathrm{CDCl}_{3}\left[\right.$ residual $\mathrm{CHCl}_{3}$ $\left(\delta_{\mathrm{H}} 7.26 \mathrm{ppm}\right), \mathrm{CDCl}_{3}\left(\delta_{\mathrm{C}} 77.0 \mathrm{ppm}\right)$ as internal standard] at $300 \mathrm{~K}$. First order chemical shifts and coupling constants were determined from one-dimensional spectra and proton resonances were assigned from COSY, TOCSY, NOESY and HETCOR experiments. Proton resonances that could not be assigned are not reported. ${ }^{13} \mathrm{C}$ resonances that originated from the fluorinated protective groups were splitt by $J_{\text {C.F }}$ coupling and therefore signals downfield from $110.0 \mathrm{ppm}$ are not reported for compound 10, 11, 12a 
and 12b. Proton decoupled gel-phase ${ }^{19} \mathrm{~F}$ NMR spectra were recorded for resin suspensions in $\mathrm{CDCl}_{3}\left[\mathrm{CFCl}_{3}\left(\delta_{\mathrm{F}} 0.00 \mathrm{ppm}\right)\right.$ as internal standard $]$ at $300 \mathrm{~K}$. Two peaks appear in the spectra at around $0.00 \mathrm{ppm}$, one resonance originates from $\mathrm{CFCl}_{3}$ inside the polymer the other resonance from $\mathrm{CFCl}_{3}$ outside the polymer. The peak with the highest chemical shift was used as internal standard. Ions for FABMS were produced by a beam of xenon atoms $(6 \mathrm{keV})$ from a matrix of glycerol and thioglycerol.

\section{2-Fluoro-4-[5-(tert-butyldiphenylsilanyloxy)-pentylselanyl]-phenol (3) tert-} Butyllitium (50 mL 1.5 M in pentane, $75.0 \mathrm{mmol}$ ) was added to a solution of 4-bromo-2fluorophenol $1(2.74 \mathrm{~mL}, 25.0 \mathrm{mmol})$ in $\mathrm{THF}(50.0 \mathrm{~mL})$ at $-78^{\circ} \mathrm{C}$. The solution was stirred for 20 minutes after which Se(s) (1.97 g, $25.0 \mathrm{mmol})$ was added in portions during $30 \mathrm{~min}$ and the mixture was allowed to reach room temperature. After stirring for additional $1 \mathrm{~h}$, the mixture was cooled to $0{ }^{\circ} \mathrm{C}$ and tert-butyl-(5-iodopentyloxy)diphenylsilane $2(11.3 \mathrm{~g}, 25.0 \mathrm{mmol})$ in THF (10.0 mL) was added during $15 \mathrm{~min}$ and the reaction was allowed to reach room temperature over night. After stirring for $15 \mathrm{~h}$ the reaction mixture was neutralized with sat. aq. $\mathrm{NH}_{4} \mathrm{Cl}(20 \mathrm{~mL})$ and the water phase was extracted with $\mathrm{CH}_{2} \mathrm{Cl}_{2}(3 \times 70 \mathrm{~mL})$. The combined organic phases was dried $\left(\mathrm{Na}_{2} \mathrm{SO}_{4}\right)$, filtrated, concentrated and purified by flash column chromatography (heptane/EtOAc, 1:8) to give $3(12.5 \mathrm{~g}, 97 \%)$ as an yellow oil; ${ }^{1} \mathrm{H}$ NMR $\left(400 \mathrm{MHz}, \mathrm{CDCl}_{3}\right) \delta 7.72(\mathrm{dd}, J=$ 7.5, $1.1 \mathrm{~Hz}, 4 \mathrm{H}, \operatorname{Ar} H), 7.49-7.38(\mathrm{~m}, 6 \mathrm{H}, \operatorname{Ar} H), 7.30(\mathrm{dd}, \mathrm{J}=10.4,1.5 \mathrm{~Hz}, 1 \mathrm{H}, \operatorname{Ar} H)$, $7.22(\mathrm{~d}, \mathrm{~J}=8.3,1 \mathrm{H}, \operatorname{Ar} H), 6.93(\mathrm{t}, J=8.7 \mathrm{~Hz}, 1 \mathrm{H}, \operatorname{Ar} H), 5.28(\mathrm{~d}, J=4.0 \mathrm{~Hz}, 1 \mathrm{H}$, $\operatorname{ArOH}), 3.69\left(\mathrm{t}, J=6.2, \mathrm{~Hz}, 2 \mathrm{H}, \mathrm{Se}\left(\mathrm{CH}_{2}\right)_{4} \mathrm{CH}_{2} \mathrm{O}\right), 2.85(\mathrm{t}, J=5.6 \mathrm{~Hz}, 2 \mathrm{H}$, $\left.\mathrm{SeCH}_{2}\left(\mathrm{CH}_{2}\right)_{4} \mathrm{O}\right), 1.69\left(\mathrm{p}, J=7.4 \mathrm{~Hz}, 2 \mathrm{H}, \mathrm{SeCH}_{2} \mathrm{CH}_{2}\left(\mathrm{CH}_{2}\right)_{3} \mathrm{O}\right), 1.59$ (p, J = 7.0 Hz, $2 \mathrm{H}$, $\left.\mathrm{Se}\left(\mathrm{CH}_{2}\right)_{3} \mathrm{CH}_{2} \mathrm{CH}_{2} \mathrm{O}\right), 1.50\left(\mathrm{~m}, 2 \mathrm{H}, \mathrm{Se}\left(\mathrm{CH}_{2}\right)_{2} \mathrm{CH}_{2}\left(\mathrm{CH}_{2}\right)_{2} \mathrm{O}\right), 1.09$ (s, $\left.9 \mathrm{H}, t \mathrm{Bu}\right) ;{ }^{13} \mathrm{C} \mathrm{NMR}$ 
$\left(100 \mathrm{MHz}, \mathrm{CDCl}_{3}\right) \delta 150.6(\mathrm{~d}, J=241.1 \mathrm{~Hz}), 142.9(\mathrm{~d}, J=14.1 \mathrm{~Hz}), 135.5,134.0,130.5$ $(\mathrm{d}, J=4.0 \mathrm{~Hz}), 129.5,127.6,120.9(\mathrm{~d}, J=18.1 \mathrm{~Hz}), 120.5(\mathrm{~d}, J=6.0 \mathrm{~Hz}), 117.7(\mathrm{~d}, J=$ 2.0 Hz), 63.7, 31.9, 29.8, 29.1, 26.9, 25.9, 19.2; ${ }^{19} \mathrm{~F}$ NMR (376 MHz, $\left.\mathrm{CDCl}_{3}\right) ; \delta-140.2$; HRMS (FAB) calcd for $\mathrm{C}_{27} \mathrm{H}_{33} \mathrm{O}_{2} \mathrm{SeSi} 516.1399 \mathrm{~m} / \mathrm{z}$, observed 516.1399 .

Ethyl 2-fluoro-4-[5-(tert-Butyldiphenylsilanyloxy)-pentylselanyl]phenoxyacetate (4) $\mathrm{K}_{2} \mathrm{CO}_{3}(1.07 \mathrm{~g}, 7.74 \mathrm{mmol})$ was added to a solution of $\mathbf{3}(2.00 \mathrm{~g}$, $3.87 \mathrm{mmol})$ in $\mathrm{CH}_{3} \mathrm{CN}(20.0 \mathrm{~mL})$ and the mixture was stirred room temperature for before a solution of ethylbromoacetate $(0.43 \mathrm{~mL}, 3.87 \mathrm{mmol})$ and $\mathrm{Bu}_{4} \mathrm{NI}(0.29 \mathrm{~g}, 0.77$ mmol) in $\mathrm{CH}_{3} \mathrm{CN}(10.0 \mathrm{~mL})$ was added during $10 \mathrm{~min}$. After stirring for $3 \mathrm{~h}$, the mixture was diluted with $\mathrm{CH}_{2} \mathrm{Cl}_{2}(150 \mathrm{~mL})$ and washed with aqueous $\mathrm{HCl}(0.05 \mathrm{M}, 3 \times 20 \mathrm{~mL})$ and brine $(3 \times 20 \mathrm{~mL})$, respectively. The organic phase was dried $\left(\mathrm{Na}_{2} \mathrm{SO}_{4}\right)$, filtrated and purified by column flash chromatography (heptane/EtOAc, 3:1) to give $\mathbf{4}(2.26 \mathrm{~g}, 97 \%)$ as a yellow oil; ${ }^{1} \mathrm{H}$ NMR $\left(400 \mathrm{MHz}, \mathrm{CDCl}_{3}\right) \delta 7.66(\mathrm{dd}, J=7.8,1.6 \mathrm{~Hz}, 4 \mathrm{H}, \mathrm{Ar} H), 7.45-$ $7.34(\mathrm{~m}, 6 \mathrm{H}, \operatorname{Ar} H), 7.27(\mathrm{~d}, J=1.8 \mathrm{~Hz}, 1 \mathrm{H}, \operatorname{Ar} H) 7.23(\mathrm{~d}, J=2.0 \mathrm{~Hz}, 1 \mathrm{H}, \operatorname{Ar} H), 7.20-$ $7.15(\mathrm{~m}, 1 \mathrm{H}, \operatorname{Ar} H), 6.81(\mathrm{t}, J=8.5 \mathrm{~Hz}, 1 \mathrm{H}, \operatorname{Ar} H), 4.66\left(\mathrm{~s}, 2 \mathrm{H}, \mathrm{COCH}_{2} \mathrm{O}\right), 4.27(\mathrm{q}, J=$ $\left.7.1 \mathrm{~Hz}, 2 \mathrm{H}, \mathrm{CH}_{3} \mathrm{CH}_{2} \mathrm{O}\right), 3.65\left(\mathrm{t}, J=6.2 \mathrm{~Hz}, 2 \mathrm{H}, \mathrm{Se}\left(\mathrm{CH}_{2}\right)_{4} \mathrm{CH}_{2} \mathrm{O}\right), 2.83(\mathrm{t}, J=7.4 \mathrm{~Hz}, 2$ $\left.\mathrm{H}, \mathrm{SeCH}_{2}\left(\mathrm{CH}_{2}\right)_{4} \mathrm{O}\right), 1.65\left(\mathrm{p}, 2 \mathrm{H}, J=7.4 \mathrm{~Hz}, \mathrm{SeCH}_{2} \mathrm{CH}_{2}\left(\mathrm{CH}_{2}\right)_{3} \mathrm{O}\right), 1.59-1.51(\mathrm{~m}, 2 \mathrm{H}$, $\left.\mathrm{Se}\left(\mathrm{CH}_{2}\right)_{3} \mathrm{CH}_{2} \mathrm{CH}_{2} \mathrm{O}\right), 1.50-1.43\left(\mathrm{~m}, 2 \mathrm{H}, \mathrm{Se}\left(\mathrm{CH}_{2}\right)_{2} \mathrm{CH}_{2}\left(\mathrm{CH}_{2}\right)_{2} \mathrm{O}\right), 1.29(\mathrm{t}, J=7.1 \mathrm{~Hz}, 3 \mathrm{H}$, $\left.\mathrm{CH}_{3} \mathrm{CH}_{2} \mathrm{O}\right), 1.05(\mathrm{~s}, 9 \mathrm{H}, t \mathrm{Bu}) ;{ }^{13} \mathrm{C} \mathrm{NMR}\left(100 \mathrm{MHz}, \mathrm{CDCl}_{3}\right) \delta 168.4,151.2(\mathrm{~d}, J=250.3$ Hz), $145.1(\mathrm{~d}, J=10.4 \mathrm{~Hz}), 135.5,134.0,129.6,129.2(\mathrm{~d}, J=3.7 \mathrm{~Hz}), 127.6,123.0$ (d, $J$ = 5.7 Hz), $121.4(\mathrm{~d}, J=18.9 \mathrm{~Hz}), 116.5(\mathrm{~d}, J=1.7 \mathrm{~Hz}), 66.8(\mathrm{~d}, J=1.0 \mathrm{~Hz}), 63.6,61.4$, 31.9, 29.8, 28.8, 26.9, 26.0, 19.2, 14.1; ${ }^{19} \mathrm{~F}$ NMR $\left(\mathrm{CDCl}_{3}, 400 \mathrm{MHz}\right) \delta$-132.7; HRMS (FAB) calcd for $\mathrm{C}_{31} \mathrm{H}_{39} \mathrm{FNaO}_{4} \mathrm{SeSi}^{+} 625.1659 \mathrm{~m} / \mathrm{z}$, observed 625.1653 . 


\section{2-Fluoro-4-[5-(tert-butyldiphenylsilanyloxy)-pentylselanyl]-phenoxyacetic}

acid (5) Aqeous $\mathrm{LiOH}(17.3 \mathrm{~mL}, 1.0 \mathrm{M})$ was added to a solution of $4(0.97 \mathrm{~g}, 1.61 \mathrm{mmol})$ in $\mathrm{THF} / \mathrm{MeOH}(10 \mathrm{~mL}, 3: 1, \mathrm{v} / \mathrm{v})$. After stirring for $3 \mathrm{~h}$ at room temperature the solution was acidified with aqueous $\mathrm{HCl}(3 \%, 100 \mathrm{~mL})$. The aqueous phase was extracted with EtOAc $(3 \times 120 \mathrm{~mL})$ and the combined organic phases were washed with brine $(100 \mathrm{~mL})$, dried $\left(\mathrm{Na}_{2} \mathrm{SO}_{4}\right)$, filtrated and evaporated to give 5 (0.93 g, quantitative) as a yellow solid; $\delta 7.65(\mathrm{dd}, J=7.9,1.5 \mathrm{~Hz}, 4 \mathrm{H}, \operatorname{Ar} H), 7.45-7.34(\mathrm{~m}, 6 \mathrm{H}, \operatorname{Ar} H), 7.25(\mathrm{~d}, J=1.7 \mathrm{~Hz}, 1 \mathrm{H}$, $\operatorname{ArH}) 7.23(\mathrm{~m}, J=2.0 \mathrm{~Hz}, 1 \mathrm{H}, \operatorname{Ar} H), 7.21-7.26(\mathrm{~m}, 1 \mathrm{H}, \operatorname{Ar} H), 6.84(\mathrm{t}, J=8.5 \mathrm{~Hz}, 1 \mathrm{H}$, $\mathrm{ArH}), 4.70\left(\mathrm{~s}, 2 \mathrm{H}, \mathrm{COCH}_{2} \mathrm{O}\right), 3.64\left(\mathrm{t}, J=6.3 \mathrm{~Hz}, 2 \mathrm{H}, \mathrm{Se}\left(\mathrm{CH}_{2}\right)_{4} \mathrm{CH}_{2} \mathrm{O}\right), 2.84(\mathrm{t}, J=7.4$ $\left.\mathrm{Hz}, 2 \mathrm{H}, \mathrm{SeCH}_{2}\left(\mathrm{CH}_{2}\right)_{4} \mathrm{O}\right), 1.66\left(\mathrm{p}, 2 \mathrm{H}, J=7.4 \mathrm{~Hz}, \mathrm{SeCH}_{2} \mathrm{CH}_{2}\left(\mathrm{CH}_{2}\right)_{3} \mathrm{O}\right), 1.55(\mathrm{p}, J=6.5$ $\left.\mathrm{Hz}, 2 \mathrm{H}, \mathrm{Se}\left(\mathrm{CH}_{2}\right)_{3} \mathrm{CH}_{2} \mathrm{CH}_{2} \mathrm{O}\right), 1.50-1.43\left(\mathrm{~m}, 2 \mathrm{H}, \mathrm{Se}\left(\mathrm{CH}_{2}\right)_{2} \mathrm{CH}_{2}\left(\mathrm{CH}_{2}\right)_{2} \mathrm{O}\right), 1.26(\mathrm{t}, J=7.1$ $\left.\mathrm{Hz}, 3 \mathrm{H}, \mathrm{CH}_{3} \mathrm{CH}_{2} \mathrm{O}\right), 1.05(\mathrm{~s}, 9 \mathrm{H}, t \mathrm{Bu}) ;{ }^{13} \mathrm{C} \mathrm{NMR}\left(100 \mathrm{MHz}, \mathrm{CDCl}_{3}\right) \delta 173.6,152.4(\mathrm{~d}, J$ $=250.2 \mathrm{~Hz}), 144.6(\mathrm{~d}, J=10.0 \mathrm{~Hz}), 135.5,134.0,129.5,129.2(\mathrm{~d}, J=3.0 \mathrm{~Hz}), 127.6$, $123.5(\mathrm{~d}, J=5.0 \mathrm{~Hz}), 121.3(\mathrm{~d}, J=19.1 \mathrm{~Hz}), 116.6,66.4,63.6,31.9,29.8,28.7,26.9$, 26.0, 19.2; ${ }^{19} \mathrm{~F} \quad \mathrm{NMR}\left(\mathrm{CDCl}_{3}, 400 \mathrm{MHz}\right) \delta \quad-132.7$; HRMS (FAB) calcd for $\mathrm{C}_{29} \mathrm{H}_{34} \mathrm{FNa}_{2} \mathrm{O}_{4} \mathrm{SeSi}^{+} 619.1165 \mathrm{~m} / z$, observed 619.1167 .

Resin 6 To a solution of $5(0.21 \mathrm{~g}, 0.36 \mathrm{mmol})$, HOAt $(0.19 \mathrm{~g}, 1.39 \mathrm{mmol})$, in DMF $3 \mathrm{~mL}$ was added DIC $(59.0 \mu \mathrm{L}, 0.47 \mathrm{mmol})$, the solution was stirred for $5 \mathrm{~min}$ and then transferred to ArgoGel- $\mathrm{NH}_{2}$ resin $(300 \mathrm{mg}, 0.12 \mathrm{mmol})$. BFB $(30 \mu \mathrm{L}, 2 \mathrm{mM}$ in DMF) was added and after $16 \mathrm{~h}$ agitation, the resin was filtrated and washed with DMF, $20 \%$ piperidine in $\mathrm{DMF},(3 \times 3 \mathrm{~mL}$ each $)$ and $\mathrm{DMF}(5 \times 3 \mathrm{~mL})$. Pyridine $(1.5 \mathrm{~mL})$ and $\mathrm{Ac}_{2} \mathrm{O}(1.5 \mathrm{~mL})$ was added to the resin and after $4 \mathrm{~h}$ agitation was the resin washed with 
DMF, $20 \%$ piperidine in DMF, $(3 \times 3 \mathrm{~mL}$ each $)$, DMF $(5 \times 3 \mathrm{~mL})$ and THF $(3 \times 3 \mathrm{~mL})$ to give resin 6; ${ }^{19} \mathrm{~F}$ NMR $\left(\mathrm{CDCl}_{3}, 400 \mathrm{MHz}\right) \delta-133.4$.

Resin 7 A solution of $\mathrm{TBAF} \times \mathrm{H}_{2} \mathrm{O}(0.19 \mathrm{~g}, 0.73 \mathrm{mmol})$ in $\mathrm{THF}(3 \mathrm{~mL})$ was added to resin $5(0.12 \mathrm{mmol})$. After $18 \mathrm{~h}$ agitation, the resin was filtrated and washed with THF $(6 \times 3 \mathrm{~mL}), 20 \%$ piperidine in DMF $(3 \times 3 \mathrm{~mL}), \mathrm{DMF}(5 \times 3 \mathrm{~mL})$ and $\mathrm{CH}_{2} \mathrm{Cl}_{2}(6 \times 3 \mathrm{~mL})$ to give resin 7 in quantitative yield; ${ }^{19} \mathrm{~F} \mathrm{NMR}\left(\mathrm{CDCl}_{3}, 400 \mathrm{MHz}\right) \delta-133.4$.

\section{2,3-Di- $O$-(4-fluorobenzoyl)-4,6- $O$-(4-fluorobenzylidene)- $\beta$-D-galactopyranose}

(9) Compound 8 (2.00 g, $3.14 \mathrm{mmol})$ and $N$-iodosuccinimide (1.41 g, $6.28 \mathrm{mmol})$ was suspended in $\mathrm{MeCN} / \mathrm{H}_{2} \mathrm{O}(40 \mathrm{~mL}, 10: 1, \mathrm{v} / \mathrm{v})$ at $0{ }^{\circ} \mathrm{C}$ in the absence of light. After stirring for $1 \mathrm{~h}$, the solution was allowed to reach room temperature and after $3 \mathrm{~h}$ was the redbrown colored solution diluted with $\mathrm{CH}_{2} \mathrm{Cl}_{2}(150 \mathrm{~mL})$ and washed with sat. aq. $\mathrm{NaHCO}_{3} / 10 \%$ aq. $\mathrm{Na}_{2} \mathrm{~S}_{2} \mathrm{O}_{3}(90 \mathrm{~mL}, 1: 1, \mathrm{v} / \mathrm{v})$. The water phase was re-extracted with $\mathrm{CH}_{2} \mathrm{Cl}_{2}(150 \mathrm{~mL})$ and the combined organic phases was dried $\left(\mathrm{Na}_{2} \mathrm{SO}_{4}\right)$, evaporated and purified by flash column chromatography (heptane/EtOAc, $2: 1 \rightarrow 1: 1$ ) to give an anomeric mixture of $9\left(0.86 \mathrm{~g},(\alpha / \beta, 1: 10,52 \%)\right.$ as a white solid; $[\alpha]^{20}{ }_{\mathrm{D}}+201^{\circ}(c 1.00$, $\left.\mathrm{CHCl}_{3}\right) ;{ }^{1} \mathrm{H}$ NMR $\left(400 \mathrm{MHz}, \mathrm{CDCl}_{3}\right) \delta$ 8.05-7.95 (m, $\left.4 \mathrm{H}, \mathrm{ArH}\right)$, 7.52-7.45 (m, $2 \mathrm{H}$, $\operatorname{Ar} H)$, 7.07-6.97 (m, $6 \mathrm{H}, \mathrm{Ar} H), 5.84(\mathrm{dd}, J=10.7,3.4 \mathrm{~Hz}, 1 \mathrm{H}, \mathrm{H}-3), 5.80-5.68(\mathrm{~m}, 2 \mathrm{H}$, H-1, H-2), 5.67 (d, $J=2.3 \mathrm{~Hz}, 0.1 \mathrm{H}, \mathrm{H}-1) 5.55$ (s, $0.1 \mathrm{H}, 4-\mathrm{FPhCHO}), 5.53(\mathrm{~s}, 1 \mathrm{H}, 4-$ FPhCHO), 5.42 (dd, $J=10.4,3.5 \mathrm{~Hz}, 0.1 \mathrm{H}, \mathrm{H}-3), 4.59$ (d, $J=3.1 \mathrm{~Hz}, 1 \mathrm{H}, \mathrm{H}-4), 4.57$ (d, $J=3.5 \mathrm{~Hz}, 1 \mathrm{H}, \mathrm{H}-4), 4.29$ (d, $J=12.3 \mathrm{~Hz}, 1 \mathrm{H}, \mathrm{H}-6), 4.17-4.07$ (m, $2.3 \mathrm{H}, \mathrm{H}-6, \mathrm{H}-5)$;

${ }^{13} \mathrm{C}$ NMR $\left(100 \mathrm{MHz}, \mathrm{CDCl}_{3}\right) \delta 100.1,100.0,95.9,91.2,74.3,73.6,72.0,71.7,69.2,68.9$, 66.7, 62.1; ${ }^{19} \mathrm{~F}$ NMR $\left(\mathrm{CDCl}_{3}, 400 \mathrm{MHz}\right) \delta-104.6(\alpha),-104.7(\alpha)-105.1(\beta),-105.2(\beta)$, 
$-112.9(\alpha)-113.3(\beta)$; HRMS (FAB) calcd for $\mathrm{C}_{27} \mathrm{H}_{20} \mathrm{~F}_{3} \mathrm{O}_{8}^{-} 529.1116 \mathrm{~m} / \mathrm{z}$, observed 529.1104.

\section{$O$-2,3-Di- $O$-(4-fluorobenzoyl)-4,6- $O$-(4-fluorobenzylidene)- $\beta$-D-}

galactopyranosyl trichloroacetimidate (10) $\mathrm{NaH}(43 \mathrm{mg}, 1.80 \mathrm{mmol})$ was added to a solution of $9(0.64 \mathrm{~g}, 1.20 \mathrm{mmol})$ in $\mathrm{CH}_{2} \mathrm{Cl}_{2} / \mathrm{Cl}_{3} \mathrm{CCN}(12 \mathrm{~mL}, 1: 1, \mathrm{v} / \mathrm{v})$ at $0{ }^{\circ} \mathrm{C}$. After $1 \mathrm{~h}$ was the mixture was allowed to attain room temperature and after $4 \mathrm{~h}$ was the mixture diluted with $\mathrm{CH}_{2} \mathrm{Cl}_{2}(150 \mathrm{~mL})$ and washed with sat. aq. $\mathrm{NaHCO}_{3}(70 \mathrm{~mL})$. The aqueous phase was re-extracted with $\mathrm{CH}_{2} \mathrm{Cl}_{2}(150 \mathrm{~mL})$ and the combined organic phases was washed with $\mathrm{H}_{2} \mathrm{O}(2 \times 100 \mathrm{~mL})$, dried $\left(\mathrm{Na}_{2} \mathrm{SO}_{4}\right)$ and evaporated to give $\mathbf{1 0}(0.83 \mathrm{~g})$ as a crude product.

\section{2,3-Di- $O$-(4-fluorobenzoyl)-4,6- $O$-(4-fluorobenzylidene)- $\beta$-D-galactopyranosyl}

fluoride (11) To a solution of $8(0.43 \mathrm{~g}, 0.72 \mathrm{mmol})$ in $\mathrm{CH}_{2} \mathrm{Cl}_{2}(15.0 \mathrm{~mL})$ and DAST $(0.14 \mathrm{~mL}, 1.10 \mathrm{mmol})$ was added NBS $(0.17 \mathrm{~g}, 0.94 \mathrm{mmol})$ at $-10{ }^{\circ} \mathrm{C}$. After stirring for 1 $\mathrm{h}$ was the solution diluted with $\mathrm{CH}_{2} \mathrm{Cl}_{2}(20 \mathrm{~mL})$ and washed with ice-cold sat. aq. $\mathrm{NaHCO}_{3}(10 \mathrm{~mL})$, sat. aq. $\mathrm{NaHCO}_{3}(50 \mathrm{~mL})$ and brine $(50 \mathrm{~mL})$. The organic phase was dried $\left(\mathrm{Na}_{2} \mathrm{SO}_{4}\right)$, evaporated and purified by flash column chromatography (heptane/EtOAc, 3:1) to give $11(0.19 \mathrm{~g}, 54 \%)$ as a white solid; $[\alpha]_{\mathrm{D}}^{20}+213^{\circ}(c 1.00$, $\left.\mathrm{CHCl}_{3}\right) ;{ }^{1} \mathrm{H}$ NMR $\left(400 \mathrm{MHz}, \mathrm{CDCl}_{3}\right) \delta$ 8.05-7.99 (m, $\left.4 \mathrm{H}, \mathrm{ArH}\right)$, 7.53-7.46 (m, $2 \mathrm{H}$, $\operatorname{Ar} H)$, 7.10-7.02 (m, $6 \mathrm{H}, \operatorname{Ar} H), 6.05(\mathrm{dd}, J=53.9,2.2 \mathrm{~Hz}, 1 \mathrm{H}, \mathrm{H}-1), 5.88-5.73(\mathrm{~m}, 2 \mathrm{H}$, H-3, H-2), 5.57 (s, $1 \mathrm{H}, 4-\mathrm{FPhCHO}$ ), 4.71 (d, $J=2.8 \mathrm{~Hz}, 1 \mathrm{H}, \mathrm{H}-4), 4.39$ (dd, $J=13.0$, $1.9 \mathrm{~Hz}, \mathrm{H}-6), 4.17-4.11$ (m, $2 \mathrm{H}, \mathrm{H}-5, \mathrm{H}-6) ;{ }^{13} \mathrm{C} \mathrm{NMR}\left(100 \mathrm{MHz}, \mathrm{CDCl}_{3}\right) \delta$ 106.6, 104.3, 100.1, 73.5, 68.7, 68.6, 68.1, 67.8, 64.6, 64.5; ${ }^{19} \mathrm{~F} \mathrm{NMR}\left(\mathrm{CDCl}_{3}, 400 \mathrm{MHz}\right) \delta$-104.7, 
$-104.8,-113.0,-149.4$; HRMS (FAB) calcd for $\mathrm{C}_{27} \mathrm{H}_{21} \mathrm{~F}_{4} \mathrm{O}_{7}^{+} 533.1218 \mathrm{~m} / z$, observed 533.1224 .

\section{Methylphenyl 2,3-di-O -(4-fluorobenzoyl)-4,6-O-(4-fluorobenzylidene)-1-}

sulfoxo- $\boldsymbol{\beta}$-D-galactopyranoside (12a and 12b) $m \mathrm{CPBA}(0.56 \mathrm{~g}, 3.24 \mathrm{mmol})$ was added to a solution of compound $8(1.04,1.63 \mathrm{mmol})$ in $\mathrm{CH}_{2} \mathrm{Cl}_{2}(10.0 \mathrm{~mL})$ at $-78{ }^{\circ} \mathrm{C}$. The mixture was allowed to reach room temperature during $8 \mathrm{~h}$ and after $23 \mathrm{~h}$ was the mixture diluted with $\mathrm{CH}_{2} \mathrm{Cl}_{2}(150 \mathrm{~mL})$ and washed with sat. aq. $\mathrm{NaHCO}_{3}(70 \mathrm{~mL})$, dried $\left(\mathrm{Na}_{2} \mathrm{SO}_{4}\right)$, evaporated and purified by flash column chromatography (heptane/EtOAc, 1:1

$\rightarrow 1: 2)$ to give the diastereomers $12 \mathbf{a}(0.65 \mathrm{~g}, 61 \%)$ and $\mathbf{1 2 b}(0.14 \mathrm{~g}, 13 \%)$ as white solids. Compound 12a had: $[\alpha]^{20}{ }_{\mathrm{D}}-20^{\circ}\left(c\right.$ 1.00, $\left.\mathrm{CHCl}_{3}\right) ;{ }^{1} \mathrm{H}$ NMR $\left(400 \mathrm{MHz}, \mathrm{CDCl}_{3}\right) \delta$ 8.00-7.87 (m, $4 \mathrm{H}, \operatorname{Ar} H), 7.74(\mathrm{~d}, J=8.1 \mathrm{~Hz}, 2 \mathrm{H}, \operatorname{Ar} H), 7.31(\mathrm{~d}, J=8.1 \mathrm{~Hz}, 2 \mathrm{H}, \operatorname{Ar} H)$, 7.11-6.95 (m, $8 \mathrm{H}, \mathrm{Ar} H), 5.72$ (t, $J=10.0 \mathrm{~Hz}, 1 \mathrm{H}, \mathrm{H}-2), 5.37$ (dd, $J=9.9,3.3 \mathrm{~Hz}, 1 \mathrm{H}$, H-3), 5.34 (s, $1 \mathrm{H}, 4-\mathrm{FPhCHO}), 4.84$ (d, $J=10.1 \mathrm{~Hz}, 1 \mathrm{H}, \mathrm{H}-1), 4.47$ (d, $J=3.1 \mathrm{~Hz}, 1 \mathrm{H}$, H-4), 4.36 (dd, $J=12.5,1.3 \mathrm{~Hz}, 1 \mathrm{H}, \mathrm{H}-6), 3.98$ (dd, $J=12.5,1.3 \mathrm{~Hz}, 1 \mathrm{H}, \mathrm{H}-6), 3.76$ (s, $1 \mathrm{H}, \mathrm{H}-5), 2.38$ (s, $3 \mathrm{H}, \mathrm{SPhMe}) ;{ }^{13} \mathrm{C} \mathrm{NMR}\left(100 \mathrm{MHz}, \mathrm{CDCl}_{3}\right) \delta 100.3,92.5,73.5,73.2$, 70.0, 68.5, 65.4, 21.6; ${ }^{19} \mathrm{~F}$ NMR $\left(\mathrm{CDCl}_{3}, 400 \mathrm{MHz}\right) \delta$-104.6, -104.7, -112.8; ( HRMS (FAB) calcd for $\mathrm{C}_{34} \mathrm{H}_{27} \mathrm{~F}_{3} \mathrm{NaO}_{8} \mathrm{~S}^{+} 675.1271 \mathrm{~m} / \mathrm{z}$, observed 675.1273.Compound 12b had: $[\alpha]^{20}{ }_{D}-25^{\circ}\left(c 1.00, \mathrm{CHCl}_{3}\right) ;{ }^{1} \mathrm{H}$ NMR $\left(400 \mathrm{MHz}, \mathrm{CDCl}_{3}\right) \delta$ 7.95-7.87 (m, $\left.4 \mathrm{H}, \mathrm{ArH}\right), 7.58$ $(\mathrm{d}, J=8.1 \mathrm{~Hz}, 2 \mathrm{H}, \operatorname{Ar} H), 7.36-7.29(\mathrm{~m}, 2 \mathrm{H}, \operatorname{ArH}), 7.16(\mathrm{~d}, J=8.1 \mathrm{~Hz}, 2 \mathrm{H}, \operatorname{Ar} H), 7.07-$ $6.97(\mathrm{~m}, 6 \mathrm{H}, \mathrm{ArH}), 5.84$ (t, $J=9.8 \mathrm{~Hz}, 1 \mathrm{H}, \mathrm{H}-2), 5.44$ (s, $1 \mathrm{H}, 4-\mathrm{FPhCHO}), 5.32$ (dd, $J$ $=10.0,3.3 \mathrm{~Hz}, 1 \mathrm{H}, \mathrm{H}-3), 4.83(\mathrm{~d}, J=9.5 \mathrm{~Hz}, 1 \mathrm{H}, \mathrm{H}-1), 4.52$ (d, $J=2.9 \mathrm{~Hz}, 1 \mathrm{H}, \mathrm{H}-4)$, $4.36(\mathrm{dd}, J=12.6,1.4 \mathrm{~Hz}, 1 \mathrm{H}, \mathrm{H}-6), 4.05$ (dd, $J=12.6,1.4 \mathrm{~Hz}, 1 \mathrm{H}, \mathrm{H}-6), 3.78$ (s, $1 \mathrm{H}$, H-5), 2.31 (s, $3 \mathrm{H}, \mathrm{SPhMe}) ;{ }^{13} \mathrm{C} \mathrm{NMR}\left(100 \mathrm{MHz}, \mathrm{CDCl}_{3}\right) \delta$ 100.0, 89.7, 73.9, 73.2, 70.2, 
68.5, 65.2, 21.4; ${ }^{19} \mathrm{~F}$ NMR $\left(\mathrm{CDCl}_{3}, 400 \mathrm{MHz}\right) \delta-104.8,-105.5,-113.1 ;$ HRMS (FAB) calcd for $\mathrm{C}_{34} \mathrm{H}_{27} \mathrm{~F}_{3} \mathrm{NaO}_{8} \mathrm{~S}^{+} 675.1271 \mathrm{~m} / z$, observed 675.1281 .

Resin 13; glycosylation with galactosyl trichloroacetimidate 10 A solution of TMSOTf (diluted 10 times with $\mathrm{CH}_{2} \mathrm{Cl}_{2}, 130 \mu \mathrm{L}, 72.0 \mu \mathrm{mol}$ ) was added to a suspension of crude product $10(0.42 \mathrm{~g}, 0.60 \mathrm{mmol})$ and resin $7(0.12 \mathrm{mmol})$ in $\mathrm{CH}_{2} \mathrm{Cl}_{2}(3.0 \mathrm{~mL})$ at room temperature. After $24 \mathrm{~h}$ agitation, the resin was filtrated and washed with $\mathrm{CH}_{2} \mathrm{Cl}_{2}$, $20 \%$ piperidine in DMF, DMF and $\mathrm{CH}_{2} \mathrm{Cl}_{2}(5 \times 3 \mathrm{~mL}$ each $)$ to give resin $\mathbf{1 3}$ in quantitative yield; ${ }^{19} \mathrm{~F}$ NMR $\left(\mathrm{CDCl}_{3}, 400 \mathrm{MHz}\right) \delta-105.0,-105.4,-113.3,-133.3$.

Resin 13; glycosylation with galactosyl fluoride 11 TMSOTf ( $65 \mu \mathrm{L}, 0.36$ mmol) was added to a suspension of compound $11(0.19 \mathrm{~g}, 0.36 \mathrm{mmol})$ and resin 7 (90 $\mu \mathrm{mol})$ in $\mathrm{CH}_{2} \mathrm{Cl}_{2} / \mathrm{Et}_{2} \mathrm{O}(4.0 \mathrm{~mL}, 3: 1, \mathrm{v} / \mathrm{v})$ at room temperature. After $19 \mathrm{~h}$ agitation, the resin was filtrated and washed with $\mathrm{CH}_{2} \mathrm{Cl}_{2}, 20 \%$ piperidine in DMF, DMF $(3 \times 4 \mathrm{~mL}$ each) and $\mathrm{CH}_{2} \mathrm{Cl}_{2}\left(6 \times 4 \mathrm{~mL}\right.$ each) to give resin 13 in quantitative yield; ${ }^{19} \mathrm{~F}$ NMR $\left(\mathrm{CDCl}_{3}\right.$, $400 \mathrm{MHz})$ 8 -105.0, -105.4, -113.3, -133.3.

Resin 13; glycosylation with galactosyl sulfoxide 12a A solution of compound 12a $(0.104 \mathrm{~g}, 0.16 \mathrm{mmol})$ in $\mathrm{CH}_{2} \mathrm{Cl}_{2}(1.6 \mathrm{~mL})$ was activated with $\mathrm{Tf}_{2} \mathrm{O}(27.0 \mu \mathrm{L}, 0.16$ mmol) at $-78{ }^{\circ} \mathrm{C}$ for $5 \mathrm{~min} .2,4,6$-collidine $(16.0 \mu \mathrm{L}, 0.12 \mathrm{mmol})$ was added and the mixture was stirred for $5 \mathrm{~min}$ before it was transferred to resin 7 (40 $\mu \mathrm{mol})$. After agitation at room temperature for $5.5 \mathrm{~h}$, the resin was filtrated and washed with $\mathrm{CH}_{2} \mathrm{Cl}_{2}$, $20 \%$ piperidine in DMF, DMF and $\mathrm{CH}_{2} \mathrm{Cl}_{2}(5 \times 3 \mathrm{~mL}$ each $)$.

Resin $14 t \mathrm{BuOOH}$ in toluene $(2 \mathrm{~mL}, 2.32 \mathrm{M})$ was added to resin $\mathbf{1 3}(40 \mu \mathrm{mol})$ and after agitation at room temperature for $4 \mathrm{~h}$, the resin was filtrated and washed with 
toluene and $\mathrm{CH}_{2} \mathrm{Cl}_{2}(5 \times 3 \mathrm{~mL}$ each $)$ to give resin 14 in quantitative yield; ${ }^{19} \mathrm{~F} \mathrm{NMR}$ $\left(\mathrm{CDCl}_{3}, 400 \mathrm{MHz}\right) \delta-104.9,-105.2,105.2,-113.2,-130.4$.

n-Pentenyl 2,3-di-O-(4-fluorobenzoyl)-4,6-O-(4-fluorobenzylidene)- $\beta$-Dgalactopyranoside (15) Toluene was added to resin $14(40 \mu \mathrm{mol})$ and after agitation at $90{ }^{\circ} \mathrm{C}$ for $8 \mathrm{~h}$, the resin was filtrated and washed with toluene $(3 \times 2 \mathrm{~mL})$. The filtrate was evaporated to give $15(22.0 \mathrm{mg}, 92 \%)$ as a white solid; $[\alpha]^{20}{ }_{\mathrm{D}}+116^{\circ}\left(\mathrm{c} 1.00, \mathrm{CHCl}_{3}\right) ;{ }^{1} \mathrm{H}$

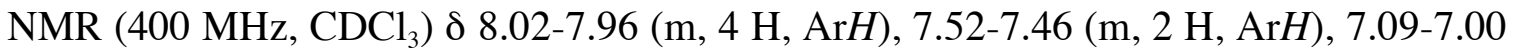
$(\mathrm{m}, 6 \mathrm{H}, \mathrm{ArH}), 5.79(\mathrm{dd}, J=10.4,8.0 \mathrm{~Hz}, 1 \mathrm{H}, \mathrm{H}-2), 5.72-5.60\left(\mathrm{~m}, 1 \mathrm{H}, \mathrm{O}\left(\mathrm{CH}_{2}\right)_{3} \mathrm{CHCH}_{2}\right)$, $5.52(\mathrm{~s}, 1 \mathrm{H}, 4-\mathrm{FPhCHO}), 5.32(\mathrm{dd}, J=10.4,3.6 \mathrm{~Hz}), 4.87-4.79(\mathrm{~m}, 2 \mathrm{H}$, $\left.\mathrm{O}\left(\mathrm{CH}_{2}\right)_{3} \mathrm{CHCH}_{2}\right), 4.72(\mathrm{~d}, J=8.0 \mathrm{~Hz}, 1 \mathrm{H}, \mathrm{H}-1), 4.56(\mathrm{~d}, J=3.6 \mathrm{~Hz}, \mathrm{H}-4), 4.39$ (dd, $J=$ 12.4, $1.5 \mathrm{~Hz}, 1 \mathrm{H}, \mathrm{H}-6), 4.13$ (dd, $J=12.4,1.5 \mathrm{~Hz}, 1 \mathrm{H}, \mathrm{H}-6), 4.01-3.94(\mathrm{~m}, 1 \mathrm{H}$, $\left.\mathrm{OCH}_{2}\left(\mathrm{CH}_{2}\right)_{2} \mathrm{CHCH}_{2}\right), 3.66$ (s, $\left.1 \mathrm{H}, \mathrm{H}-5\right), 3.56-3.49$ (m, $\left.1 \mathrm{H}, \mathrm{OCH}_{2}\left(\mathrm{CH}_{2}\right)_{2} \mathrm{CHCH}_{2}\right), 2.05-$ $1.91\left(\mathrm{~m}, 1 \mathrm{H}, \mathrm{O}\left(\mathrm{CH}_{2}\right)_{2} \mathrm{CH}_{2} \mathrm{CHCH}_{2}, 1.63\left(\mathrm{~h}, J=7.2 \mathrm{~Hz}, \mathrm{OCH}_{2} \mathrm{CH}_{2} \mathrm{CH}_{2} \mathrm{CHCH}_{2}\right) ;{ }^{13} \mathrm{C} \mathrm{NMR}\right.$ $\left(100 \mathrm{MHz}, \mathrm{CDCl}_{3}\right) \delta 101.1,100.3,73.6,72.8,69.3,68.9,68.9,66.4,29.8,28.5 ;{ }^{19} \mathrm{~F} \mathrm{NMR}$ $\left(\mathrm{CDCl}_{3}, 400 \mathrm{MHz}\right) \delta-105.0,-105.7,-113.3$; HRMS (FAB) calcd for $\mathrm{C}_{32} \mathrm{H}_{29} \mathrm{~F}_{3} \mathrm{NaO}_{8}{ }^{+}$ $621.1707 \mathrm{~m} / \mathrm{z}$, observed 621.1704. 